\title{
Mechanical Design of the VISA Undulator
}

\author{
M. Libkind \\ L. Bertolini \\ P. Duffy \\ R. Carr \\ G. Rakowsky \\ J. Skaritka
}

This paper was prepared for submittal to the

Particle Accelerator Conference 1999

New York, NY

March 27 - April 4, 1999

March 1999

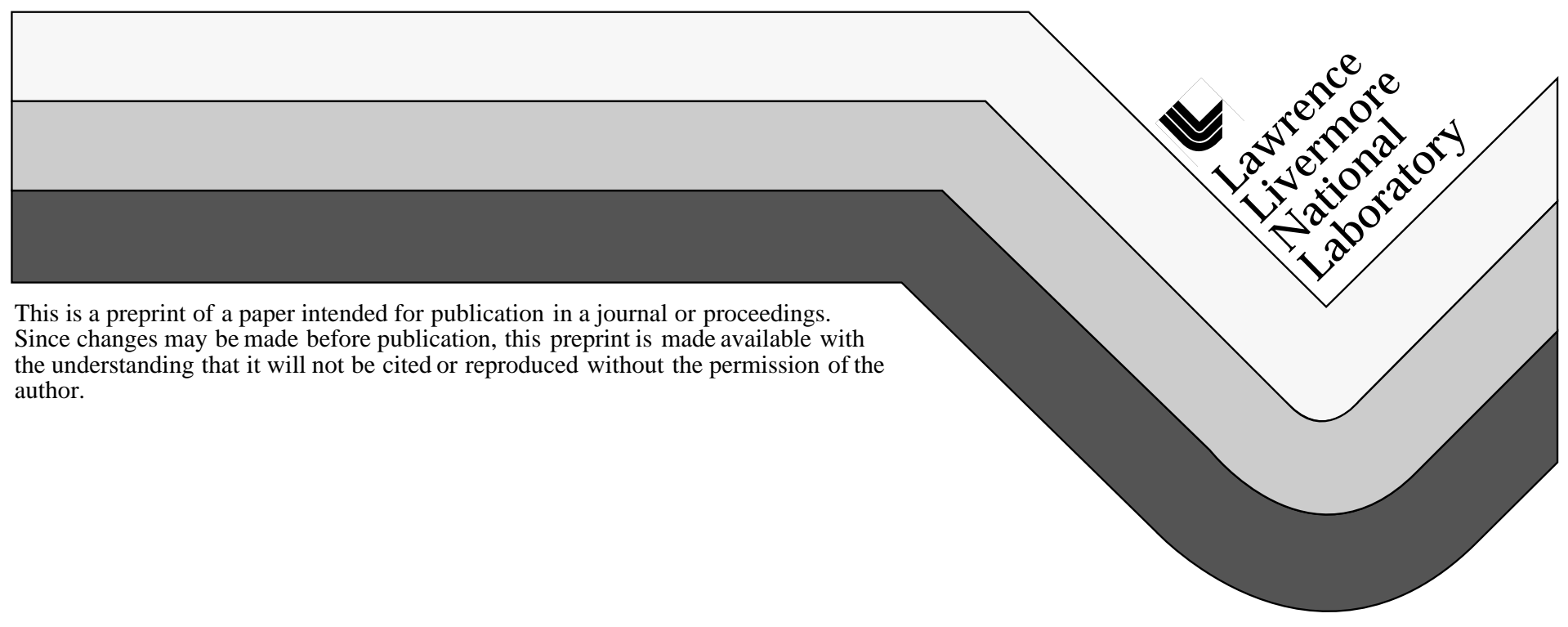




\section{DISCLAIMER}

This document was prepared as an account of work sponsored by an agency of the United States Government. Neither the United States Government nor the University of California nor any of their employees, makes any warranty, express or implied, or assumes any legal liability or responsibility for the accuracy, completeness, or usefulness of any information, apparatus, product, or process

disclosed, or represents that its use would not infringe privately owned rights. Reference herein to any specific commercial product, process, or service by trade name, trademark, manufacturer, or otherwise, does not necessarily constitute or imply its endorsement, recommendation, or favoring by the United States Government or the University of California. The views and opinions of authors expressed herein do not necessarily state or reflect those of the United States Government or the University of California, and shall not be used for advertising or product endorsement purposes. 


\title{
MECHANICAL DESIGN OF THE VISA UNDULATOR
}

\author{
M. Libkind $^{1}$, L. Bertolini, P. Duffy, LLNL, Livermore, CA 94550 \\ R. Carr, SLAC, Stanford, CA 94309 \\ G. Rakowsky, J. Skaritka, BNL, Upton, NY 11973
}

\begin{abstract}
As part of the research and development effort for a 4th generation light source, we have designed a 4-meter long Free-Electron Laser (FEL) undulator. The undulator will be installed at the Accelerator Test Facility (ATF) at Brookhaven National Laboratory to conduct a SelfAmplified Spontaneous Emission (SASE) demonstration. The demonstration is called VISA, which stands for "Visible to Infrared SASE Amplifier." The undulator consists of 440 permanent dipole magnets per meter which are supported and aligned on a precision strongback. Focusing and defocusing permanent quadrupole magnets are also supported by the strongback. Each of the 4 one-meter sections of undulator are kinematically supported and housed within the vacuum vessel. The undulator and the vacuum vessel are supported independently to eliminate undulator misalignment during vacuum pump-down of the vessel. We describe the design requirements and features of the undulator, vacuum vessel and support system.
\end{abstract}

\section{INTRODUCTION}

SLAC, ANL, LANL, LLNL and UCLA are proposing to build a Free-Electron Laser (FEL) R\&D facility operating in the self-amplified spontaneous emission (SASE) mode in the wavelength range 1.5-15 $\AA$. This FEL, called "Linac Coherent Light Source" (LCLS) [1], utilizes the SLAC linac and produces sub-picosecond pulses of short wavelength $\mathrm{x}$-rays with very high peak brightness and full transverse coherence. The Visible to Infrared SASE Amplifier to be installed at the Accelerator Test Facility (ATF) at BNL was designed to demonstrate SASE through saturation.

The critical component of the experiment is the 990millimeter long undulator segment. VISA includes four of these segments, but the design, including vacuum vessel, was based on the need to extend the undulator to six meters for use at BNL in the Source Development Laboratory (SDL) upon the completion of the VISA experiment.

Significant challenges included a dipole gap tolerance of 10 microns with a profile of 25 microns per meter, absolute axial dipole positioning of 25 microns, vertical quadrupole positioning of 50 microns and a quadrupole gap tolerance of 50 microns.

\section{UNDULATOR}

Figure 1 shows the cross-section of the VISA undulator and Figure 2 shows the undulator with top strongback removed. The strongbacks and keepers were fabricated

\footnotetext{
${ }^{1}$ E-mail: libkind1@1lnl.gov
}

using a combination of grinding and milling in order to obtain the required 25-micron profile.

Left and right keeper assemblies are precision mounted to the ground bottom strongback. The quadrupole holder assemblies (described below) are installed. The initial alignment of the holders is obtained using a temporary 1-meter long ground spacer bar. Threaded rods passing through the keepers hold the quadrupole holders in place and permit horizontal trajectory adjustment after assembly is complete.

The top 220 dipoles are then mounted using a precision installation mechanism and hold-down bars to obtain the required axial positioning.

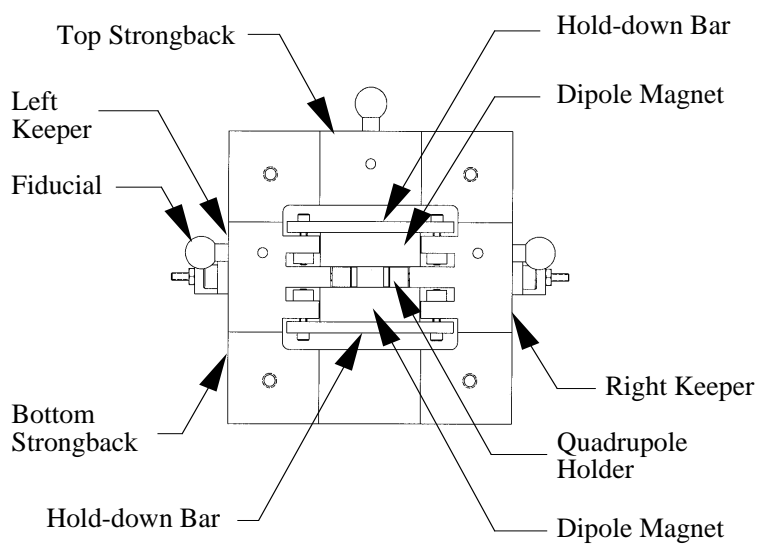

Figure 1. Cross-section of the VISA undulator

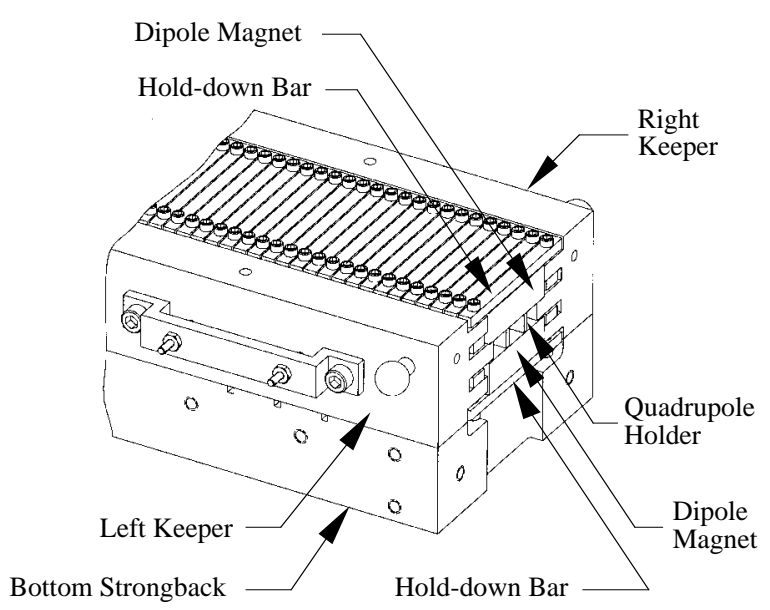

Figure 2. Undulator with top strongback removed

Next the top strongback is attached and the bottom strongback is removed. The remaining 220 dipole magnets are installed and the bottom strongback is re-attached. 
After assembly a 1-mil nickel foil was installed to cover the dipole magnets in order to provide a better RF environment.

Built into the keepers are holes that permit insertion of diagnostic probes into the beam at the undulator segment quarter points.

Each left and right focusing or defocusing quadrupole section consists of three 30-millimeter long magnets that are mounted in a holder assembly (Figure 3 ). Physical constraints limit the thickness of the back of the holder and the thickness of the holder faceplate to 1.5 millimeters each. However, the clamping of the magnets between the holder and the faceplate creates a stiff laminated structure.

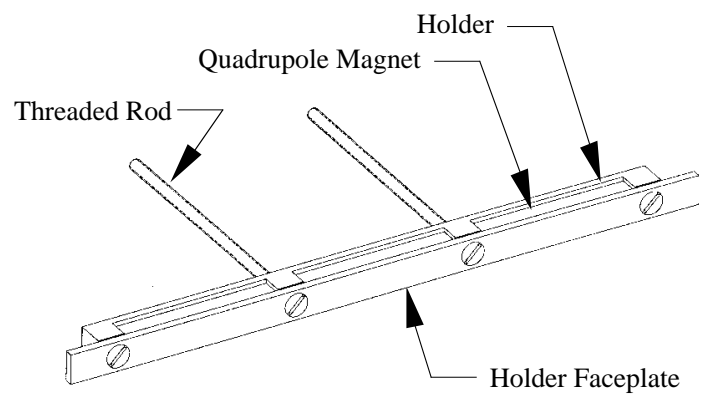

Figure 3. Quadrupole holder assembly

The straightness of the three quadrupole magnets in a section is obtained by assembling the holder against a precision ground plate with temporary shims to locate the magnets vertically. The height of the holder is controlled so that it is self-positioning between the top and bottom dipoles. The final design allows the maximum deviation of a quadrupole section from true vertical center to be 100 microns.
Fiducials are located at the ends of each undulator segment to facilitate alignment in ATF. The magnetic field is shimmed and the magnetic center of each undulator determined relative to the fiducials in the NSLS Magnetic Measurements Laboratory at BNL. To accomplish this, built into the each undulator is the ability to rotate it 90 degrees around the beam axis with negligible sag in the structure.

The ends of the undulator segments are designed to accept the attachment of termination magnets in a holder. These are used during magnetic shimming of each segment and on the upstream and downstream ends of the entire undulator during operation.

\section{VACUUM VESSEL}

The vacuum vessel for VISA must house the undulator, while remaining mechanically de-coupled from it, and support the diagnostic probes. It must also allow installation access and maintain vacuum integrity. Figure 4 is an isometric view of the vessel and support system.

The four-meter VISA vacuum vessel is constructed in two sections; one 1.5 meters and one 2.5 meters long. This permits installation in ATF and expansion of the vessel to six meters in SDL. The expansion is accomplished by installing a 2 -meter vessel section between the 1.5 and 2.5-meter sections.

The cross-section of the vacuum vessel is limited to $203 \times 203$ millimeters by the dimensions of the external steering coils although access ports and diagnostics protrude beyond. Each coil has a removable top section that permits removal of the vessel covers. Ports on the top and one side of the vessel permit access to the fiducials on the undulator sections during alignment.

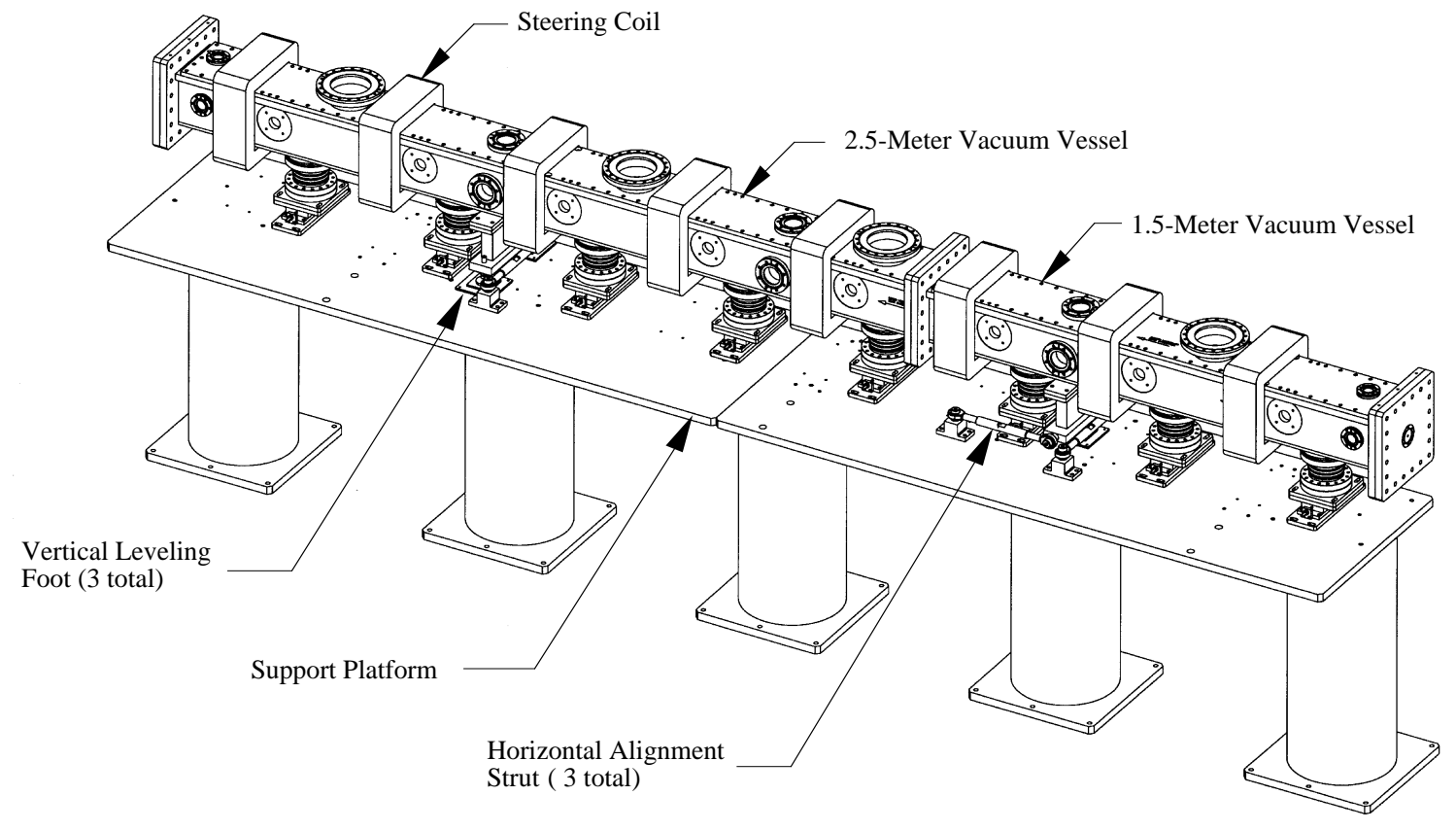

Figure 4. VISA vacuum vessel and support system 
Finite element analysis was used to calculate the deformation of the vacuum vessel under gravity and vacuum loading. Vertical deformation of the vessel under these loads is within acceptable limits. Removable shoulder bolts through the vessel covers and top flanges restrain the vessel sidewalls from bending inward during pump-down. This is critical because the insertable diagnostic probes are attached to the vessel sidewalls. Shoulder bolts are used instead of specifying a required torque on the many cover bolts thereby reducing the vessel closing time.

Ports for the insertable diagnostic probes are located at the quarter points of the undulator segments. The probes are aligned to corresponding holes in the undulator keepers at atmospheric pressure. Oversize clearance holes in the mounting flanges provide the necessary vertical and horizontal adjustment range.

Fiducials are located at the ends of the vessel to facilitate alignment, which need only be on the order of one millimeter.

The vacuum vessel is supported on three leveling feet with reinforced Teflon pads. Two transverse and one axial struts control the horizontal positioning.

\section{SUPPORT SYSTEM}

The support platform consists of two aluminum plates and five cylindrical aluminum piers. The fundamental frequency of the support platform, vacuum vessel and undulators is $20 \mathrm{~Hz}$. The plate also supports alignment hardware during installation.

Figure 5 shows the support system for the undulator segments. Each undulator is supported kinematically on three vertical adjuster screws at approximately the quarter points. A ball, free to rotate, forms the tip of each adjuster. The adjusters are connected to support pedestals that pass through the bottom of the vacuum vessel and attach to slide mechanisms that permit the adjusters to be moved perpendicular to the beam axis. Vacuum integrity of the vessel is obtained using a welded bellows between the vessel and the blank flange located on each slide.

Axial positioning of one of the undulator segments is obtained by fixing one of its support balls in a cone; this forms the axial reference for the entire four meters. The remaining three undulator segments are fixed axially to the reference through a spring and adjuster mechanism. This design permits axial spacing of the undulator segments within less than the maximum thermal expansion.

\section{SUMMARY}

The design of the VISA undulator, vacuum vessel and support system has been described. The hardware is currently being assembled at BNL in the Accelerator Test Facility.

\section{ACKNOWLEDGMENTS}

The work of M. Libkind, L. Bertolini and P. Duffy was supported by the Office of Energy Research, Office of High Energy and Nuclear Physics Division, of the U.S. Department of Energy under contracts, DE-AC0376SF00098 and W-7405-ENG-48-(LLNL). The work of R. Carr was supported by the U.S. Department of Energy, Office of Basic Energy Sciences under contract number DE-AC03-76SF00515. The work by G. Rakowsky and J. Skaritka was supported by U.S. Department of Energy under contract DE-AC02-98CH10886.

\section{REFERENCES}

[1] Linac Coherent Light Source (LCLS) Design Study Report, SLACR-521, UC-414, April 1998.

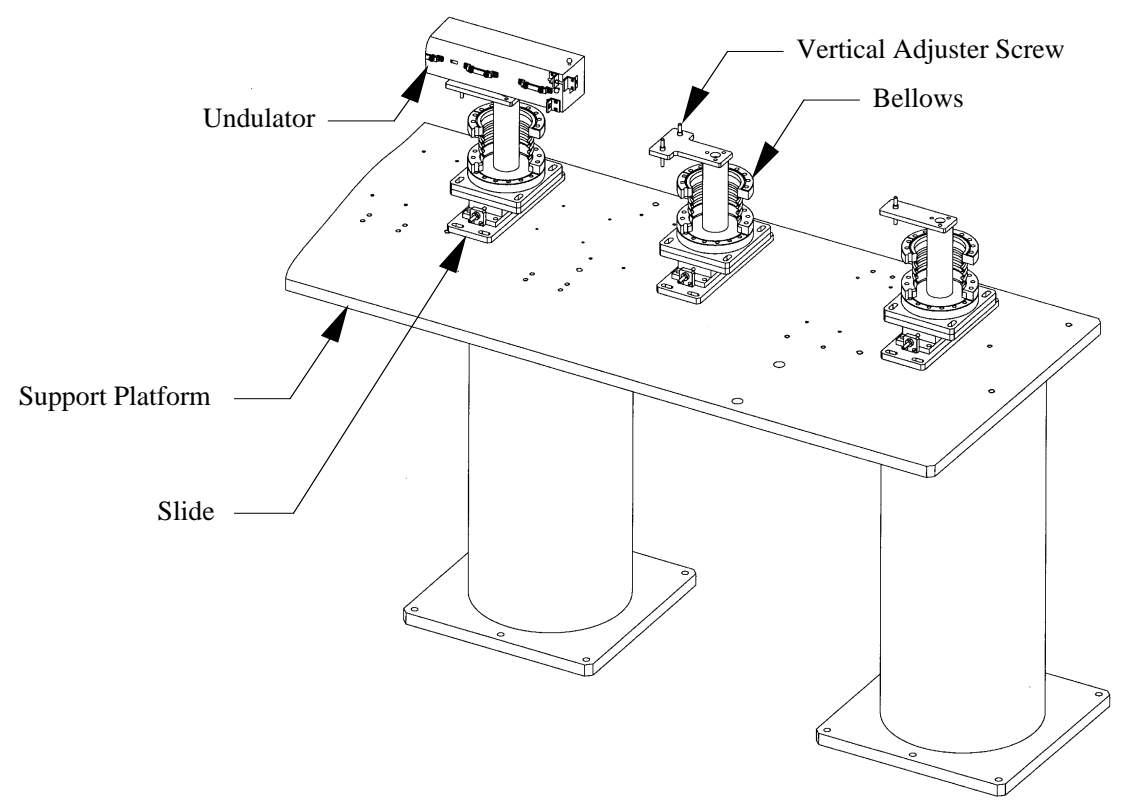

Figure 5. Support system for undulator segments 